\title{
Diverse manifestations of obstructive sleep apnoea in pregnancy at a Chinese hospital
}

\begin{abstract}
OSA is a common disorder in the general adult population. There are several studies linking OSA symptoms with fetal and maternal complications including gestational hypertensive disorders, gestational diabetes and possibly preterm labor. However, its prevalence in pregnant women remains unknown, mostly because obstetrical care providers do not appropriately screen for the disorder. During the past years, we have paid attention to the disease. Here we will discuss the typical presentations of OSA but also report some less obvious presentations to help providers recognize those manifestations and screen for the disorder when warranted. Our case series describes patients with diagnoses such as chronic hypertension, pre-eclampsia, left atrium hypertrophy, gestational diabetes mellitus and nocturnal dyspnea, or with OSA family history, who were diagnosed with OSA and offered treatment with CPAP during pregnancy.
\end{abstract}

Keywords: obstructive sleep apnoea, pregnancy, gestational hypertensive disorders, continuous positive airway pressure
Volume 2 Issue 2 - 2017

\author{
Jun Wei,' He Cai,' Lili Xu, ${ }^{2}$ Qi Xu,' Yan \\ Wang,' Guoli Liu' \\ 'Department of Obstetrics \& Gynecology, Peking University \\ People's Hospital, China \\ ${ }^{2}$ Department of Obstetrics \& Gynecology, People's Hospital of \\ Anshun, China
}

\begin{abstract}
Correspondence: Guoli Liu, People's Hospital Peking University, No II Xizhimen South Street, Xicheng District, Beijing, China, Tel: | 366 | 0 | 4583; Fax +86- |0-88324870, Email liuguoli@pkuph.edu.cn \#the authors contribute equally to this paper
\end{abstract}

Received: March 28, 2017 | Published: April 27, 2017
Abbreviations: SDB, sleep disordered breathing; OSA, obstructive sleep apnea; CPAP, continuous positive airway pressure; PCOS, polycystic ovary syndrome; BMI, body mass index; TST, total sleep time; PSG, Polysomnography; AHI, apnea hypopnea index; REM, rapid eye movement sleep; GDM, gestational diabetes mellitus

\section{Introduction}

Sleep disordered breathing (SDB) refers to a group of disorders characterized by abnormal respiratory patterns (e.g., apneas, hypopneas) or abnormal gas exchange (e.g., hypoxia) during sleep. Obstructive sleep apnea (OSA), the most common type of SDB, is characterized by airway narrowing during sleep that leads to respiratory disruption, hypoxia, and sleep fragmentation. ${ }^{1,2}$ Classic symptoms of OSA include snoring, gasping, witnessed apnoea, daytime sleepiness and fatigue. Basic physical examination involves measurement of height, weight and body mass index (BMI), as well as an evaluation of upper airway patency, including the a small mandible and neck circumference. In the non-pregnant population, OSA is associated with chronic hypertension, ${ }^{3,4}$ type II diabetes and measures of abnormal glucose metabolism. ${ }^{5,6}$

Many studies have demonstrated that SDB symptoms (snoring, excessive daytime sleepiness) are common in pregnancy and that the prevalence of SDB symptoms increases as pregnancy progresses..$^{7-10}$ this progression is at least partly related to the weight gain, edema and hyperemia of pregnancy that lead to upper airway narrowing and increased airway resistance. Pregnant women with loud snoring were found to be at increased risk of developing pre-eclampsia ${ }^{11,12}$ and gestational diabetes. ${ }^{11,13}$ Continuous positive airway pressure (CPAP) is the most effective non-invasive treatment of OSA and has been used successfully in pregnancy. ${ }^{14}$

During the past years, we have paid some attention to the relationship of OSA and hypertensive disorders in pregnancy (including chronic hypertension, hypertension in pregnancy and pre-eclampsia). Our data also suggested snoring was an independent risk factor of developing pre-eclampsia. ${ }^{15}$ Due to studies addressing OSA's impact on pregnancy are heterogeneous in regards to study design and OSA definition, it is premature to suggest that obstetrical care providers should systematically screen for OSA in pregnancy. ${ }^{16}$ But we should pay attention to the high risk pregnant women of this disease. Interestingly, beyond the most typical symptoms of snoring and witnessed apneas, there are some clinical manifestations of the disorder. The patients discussed in this series have been selected from a database of pregnant women with polysomnographically diagnosed OSA. Here we will discuss the diverse clinical manifestations of obstructive sleep apnoea in pregnancy.

\section{Case reports}

\section{Case I}

A 38-year-old graivida 3 para 0 , admitted to hospital at 36weeks and 5days'gestation with severe chronic hypertension. She had taken regular medication for 10years with a diagnosis of chronic hypertension. At the pregnancy onset, anti-hypertensive was changed to labetalol $100 \mathrm{mg}$ three times daily and amlodipine besylate $2.5 \mathrm{mg}$ daily. The past history included polycystic ovary syndrome (PCOS) and diabetes mellitus for 2years. Her body mass index (BMI) was $34.2 \mathrm{~kg} / \mathrm{m}^{2}$ prior to pregnancy and $37.5 \mathrm{~kg} / \mathrm{m}^{2}$ at the current presentation. Echocardiography displayed left atrium hypertrophy and Left ventricular ejection fraction is $63 \%$.

A diagnostic polysomnography (PSG) was performed at 19thweeks of gestation due to snoring, obese, hypertension and diabetes mellitus. The apnea hypopnea index (AHI) was 51per hour. A number of episodes of desaturation were observed, the minimum oxygen saturation $\left(\mathrm{SaO}_{2}\right)$ was $79 \%$ and oxygen desaturation index (ODI) was $9.5 / \mathrm{h}$. When the patient was diagnosed with severe obstructive sleep apnea (OSA), treatment with continuous positive airway pressure (CPAP) (AutoSet ${ }^{\mathrm{TM}}$, ResMed, and Sydney, Australia) 
was introduced. Nearly 4weeks later, it was reported that the snoring symptoms had been greatly resolved. CPAP therapy resulted in a significant reduction of oxygen desaturation. The mean $\mathrm{SaO}_{2}$ was $97 \%$, the minimum $\mathrm{SaO}_{2}$ was increased to $92 \%$ and ODI diminished to $0.3 / \mathrm{h}$ on room air breathing. However, mean blood pressure was not greatly improved by CPAP (BP $160 / 100 \mathrm{mmHg}$ on admission). The patient had a caesarean section at 36 weeks and 5 days for persistent Table I Demographics and neonatal outcomes in 6 patients with OSA fetal decelerations and delivered a female infant with Apgar scores of 9 and 10. Placental pathology showed accelerated villous maturity, capillary congestion, focal per villous fibrin deposition and decadal vasculopathy. CPAP therapy was declined after birth and the nocturnal snoring returned with discontinuation of the treatment. Neonatal outcomes are detailed in Table 1.

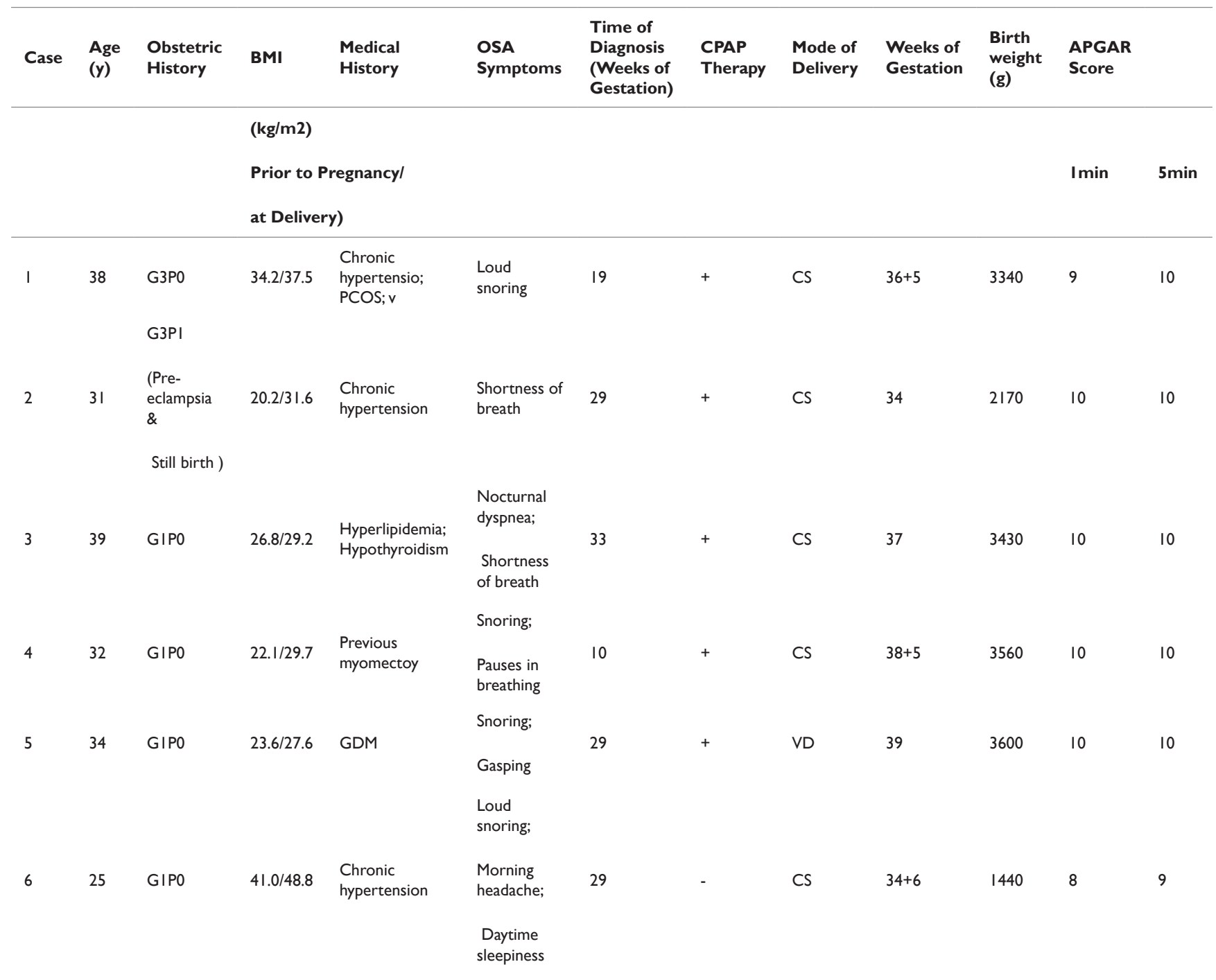

OSA, Obstructive sleep apnea, PCOS, Polycystic Ovary Syndrome; DM, Diabetes mellitus; GDM, Gestational Diabetes Mellitus; CPAP, Continuous Positive Airway Pressure; CS, Caesarean Section;VD, Vaginal Delivery

\section{Case 2}

A 31-year-old woman, G3P1, presented at 34weeks of gestation for severe abdominal pain. Her obstetrical history was significant for severe preeclampsia and still birth in her previous pregnancy. She developed chronic hypertension and 24-h ambulatory blood pressure monitoring revealed mild nocturnal hypertension. Prior to pregnancy, her weight was $53 \mathrm{~kg}$ and BMI $20.2 \mathrm{~kg} / \mathrm{m}^{2}$.

As the patient reported nocturnal hypertension, shortness of breath during sleep, she underwent PSG at 29 weeks. PSG findings were as follows: total sleep time (TST) 379min, rapid eye movement sleep (REM) 90 min. The absolute number of respiratory events during sleep was 190(7 central, 5 obstructive and 0 mixed apneas, and 17 hypopneas) and the AHI was 30.1 (severe OSAS), with a mean $\mathrm{SaO} 2$ $96.5 \%$, a minimum $\mathrm{SaO}_{2} 84.6 \%$ and ODI 12.2/h. She started CPAP at around 29 weeks of gestation and gasping symptom was completely resolved but BP not greatly improved.

There was tenderness in the lower abdomen. Ultrasonography showed that placental location was "anterior" with vast sub chorionic hypoechogenic area interpreted as a sign of abruption placenta. Fetal heart rate at ultrasound examination was $100-110$ beats per minute. She was delivered of a 34 weeks old premature baby weighing $2170 \mathrm{~g}$ by emergency cesarean. The placenta showed a large retro placental 
clot. Placental pathology showed villous stoma hemorrhage and few scattered neutrophils in the chorionic space. The infant stayed at the neonatal intensive care unit for 2 months and was found to be doing well without major complications at 6 month postoperative follow-up.

\section{Case 3}

A39-year-old women (G1P0) at 33weeks gestation visited in our center with a chief complaint of nocturnal dyspnea and episodes of shortness of breath that arouse from sleep. The past history included hyperlipidemia for 10 years and hypothyroidism for 7years. Vital signs included a body mass index BMI of $26.8 \mathrm{~kg} / \mathrm{m}^{2}$ and blood pressure of $110 / 75 \mathrm{mmHg}$. The cardiopulmonary examination was unremarkable. Parameters of electrocardiogram and echocardiogram were within normal limits. Due to the complaint of nocturnal dyspnea, obstructive sleep apnea (OSA) was then suspected. PSG revealed an apnea-hypopnea index (AHI) of $5 / \mathrm{h}$, lowest saturation had a value of $89 \%$. The test revealed a number of episodes of obstructive apnea and hypopnea. The patient was diagnosed with mild obstructive sleep apnea and then CPAP was given. The used of CPAP allowed a reduction of the AHI to $0.7 / \mathrm{h}$. Sleep quality was much improved and nocturnal symptoms were completely resolved. The patient delivered a live $3430 \mathrm{~g}$ healthy female infant by elective caesarean section for breech presentation at 37 thweeks of gestation (Table 1).

\section{Case 4}

A 32-year-old woman was admitted to the ward at term for induction of labor. Medical history included a previous myomectomy. Family history was remarkable for hypertension and OSA in her mother. The patient reported that she snored terribly all over the night and that several pauses in her breathing were observed by her husband at night. Diagnostic PSG demonstrated the absolute number of respiratory events during sleep was 180 (171 obstructive apneas, and 9 hypopneas) and an AHI of $23 / \mathrm{h}$, with the mean oxygen saturation of $87 \%$. The patient was started on CPAP treatment around 10 weeks' of gestation. Symptomatic improvement began approximately one month after initiation of CPAP. The mean $\mathrm{SaO}_{2}$ was $97 \%$, the minimum $\mathrm{SaO}_{2}$ was increased to $93 \%$ and ODI diminished to $0.38 / \mathrm{h}$ at 23 weeks gestation. At 38 weeks and 5days' of gestation, caesarean section was performed for scarred uterus. Though further CPAP treatment was strongly recommended, it wasn't continued after discharging from hospital. During the follow up period, the patient reported impairments in memory and learning ability, which were related to the aforementioned snoring symptoms and the infant was also suffered from loud snoring.

\section{Case 5}

A 34- year-old pregnant women, was diagnosed with Gestational Diabetes Mellitus (GDM) at 24weeks of gestation. She was followed in our clinic until an elective delivery was planned for 39 weeks. Baseline physical characteristics were as follows: height, $162 \mathrm{~cm}$; weight prior to pregnancy $62.0 \mathrm{~kg} ; \mathrm{BMI}, 23.6 \mathrm{~kg} / \mathrm{m}^{2} ; \mathrm{BMI}$ at time of vaginal delivery $27.6 \mathrm{~kg} / \mathrm{m}^{2} ; \mathrm{BP}, 118 / 78 \mathrm{mmHg}$; The cardiopulmonary examination was unremarkable. Labor was induced with a vaginal prostaglandin E2 (PGE2) insert. A 3600g male infant was delivered successfully.

The patient had been snoring for nearly 10years without any other notable medical history. Her husband had complained about her snoring, and she used to hold his breath during sleep and subsequently needed to gasp for breath. Frequent loud snoring and other respiratory events were observed particularly when she was sleeping in a supine position. Her other complaint was fatigue beginning during the previous weeks. The PSG was performed at 29 weeks of gestation and the test revealed an AHI score of 89.7 and lowest oxygen saturation of $60 \%$, which was consistent with a diagnosis of severe OSA. She was treated with CPAP therapy throughout the remainder of her pregnancy and 5days after the initial CPAP, the mean $\mathrm{SaO}_{2}$ was increased to $97 \%$, the minimum $\mathrm{SaO}_{2}$ was $73 \%$ and ODI was $14 / \mathrm{h}$. She noted that nocturnal snoring was much resolved. Follow-up visit demonstrated that stop using the device after discharging. The patient reported mild improvement in symptom of snoring, but she still felt fatigue in the daytime. Repeat polysomnography was declined.

\section{Case 6}

A 25-year-old women presented in our center during her first pregnancy, at 29 weeks of gestation. Her medical history was unremarkable except for a previous diagnosis of hypertension without taking medication. The patient was admitted to hospital with high blood pressure, proteinuria and increasing edema. Physical examination revealed obesity $\left(\mathrm{BMI} 41 \mathrm{~kg} / \mathrm{m}^{2}\right.$ ) and blood pressure $160 / 110 \mathrm{mmHg}$. The sleep focused interview revealed typical OSA symptoms, which had increased as her pregnancy progressed: severe snoring, morning headache, sleep daytime sleepiness. PSG showed the minimum $\mathrm{SaO}_{2}$ was $84 \%$ and ODI was as high as $8.8 / \mathrm{h}$. Despite strong recommendations, the patient refused CPAP due to concerns of claustrophobia. At 34weeks and 4days, severe oligohydramnios was observed (the amniotic fluid index decreased to $3.6 \mathrm{~cm}$ ). The patient was diagnosed with pre-eclampsia superimposed on chronic hypertension and delivered a live $1440 \mathrm{~g}$ male infant by caesarean section. The baby boy was diagnosed with congenital biliary atresia and presented with jaundice at 6-months old. Unfortunately, the child died at 7-months old. Till now, this patient hasn't gotten pregnancy again.

\section{Comment}

OSA is a common disorder in the general adult population. It remains under-diagnosed due to the lack of awareness of the disease by patients and the lack of screening by physicians. OSA is diagnosed in $10-15 \%$ of women in the general population, but its prevalence in pregnant women remains unknown. The incidence of symptoms of sleep disordered breathing has been reported in European and North American studies and shows that snoring affects $14-41 \%$ of pregnant women ${ }^{11,12}$ and is more common than in non-pregnant controls. ${ }^{5,6,17,18}$ Our data also showed that incidence of snoring in the 2nd Trimester or 3 rd trimester was higher than that in 1st trimester or before pregnancy, the figure was $20.13 \%, 24.46 \%$ vs $5.63 \%, 5.41 \%$ independently. ${ }^{15}$ This change might be at least partly related to the weight gain, edema and hyperemia of pregnancy that lead to upper airway narrowing and increased airway resistance.

Clinical consequences of OSA include excessive daytime somnolence, morning headaches, daytime fatigue and possibly cognitive impairment. Partners usually report loud cyclical snoring, gasping, witnessed apneas, restlessness and thrashing movements of the extremities during sleep. Women tend to report snoring less commonly than men do. Personality changes, work-related problems including poor judgement, depression and intellectual deterioration may also be observed. Strong associations of OSA co-existing with chronic HTN have been reported, however, more recent reports on the development of new onset HTN seem to be conflicting. ${ }^{3,4}$ Associations 
of OSA with type II diabetes are also reported ${ }^{5,6,17}$ with abnormalities in glucose metabolism found independently of body habits. Also the association of OSA and gestational diabetes mellitus was found, but not with preeclampsia, preterm birth or fetal growth. ${ }^{16}$

Among this six case series, three had chronic HTN, two of the three patients were obese with BMI $34.2,41 \mathrm{~kg} / \mathrm{m}^{2}$ respectively, the other one suffered from nocturnal hypertension, shortness of breath during sleep, and OSA was diagnosed at 19, 29, 29 gestational weeks. Here it is obvious that the strong associations of OSA co-existing with chronic HTN. With the elder pregnant women increased, our unpublished data manifested that percentage of chronic HTN in the total pregnancy at Peking University People's Hospital increased too, from $1.56 \%$ in $2014 y, 2.36 \%$ in $2015 y$ to $2.94 \%$ in $2016 y$. So for such chronic HTN pregnant women, the obstetrical care providers should systematically screen for SDB from the first trimester even earlier to the pre-pregnant time. Apart from the chronic HTN, the chief complaints of the remaining three patients included nocturnal dyspnea and episodes of shortness of breath that arouse from sleep, severe snoring with OSA family history and ten years snoring accompanied with gestational diabetes mellitus in pregnancy. We had a pity for the above three patients, it would be better for them to be diagnosed earlier due to the chronic severe snoring. For the fourth case, during the postpartum follow-up, the patient reported impairments in memory and learning ability, which were related to the aforementioned snoring symptoms and her infant was also suffered from loud snoring. Her family OSA history should be further explored to learn the true etiology in such a family.

CPAP is the most effective non-invasive form of therapy for OSA. Yet, in non-pregnant adults, the role of CPAP in reversing or preventing long-term cardiovascular and metabolic morbidity remains unanswered, especially for patients with only mild OSA. ${ }^{19-22}$ Similarly; the role of CPAP in preventing maternal/fetal morbidity during pregnancy has yet to be determined. In Table 2, we listed the change of oxygen saturation with CPAP treatment for these OSA patients. Significant improvement of oxygen saturation was observed. Large, prospective cohorts that use objective OSA assessments across pregnancy are needed to accurately define the impact of OSA on pregnancy outcomes. Secondly, clinical trials of CPAP use in pregnancy are needed to determine if short term treatment of OSA in pregnancy can improve maternal and neonatal health.

In summary, OSA may have a variety of clinical presentations and may have immediate consequences on cognitive function as well as long-term consequences such as poor cardiovascular outcomes and learning ability. For the obstetrical care providers, various symptoms of OSA and sleep disordered breathing should be recognized and a clinical assessment of pregnant patients with known associated co morbidities such cardiovascular risk factors and obesity needs to occur.

Table 2 Polysomnographic and Oxygen saturation data of the 6 patients

\begin{tabular}{llllll}
\hline \multirow{2}{*}{ Case } & Prior to cpap & \multicolumn{5}{l}{ Post to cpap } \\
\cline { 2 - 6 } & $\mathbf{A H I}($ Per Hour) & Minimum $\mathrm{SaO}_{2}(\%)$ & Mean $\mathrm{SaO}_{2}(\%)$ & Minimum $\mathrm{SaO}_{2}(\%)$ & ODI (Per Hour) \\
\hline 1 & 51 & 79 & 97 & 92 & 0.3 \\
2 & 30.1 & 84.6 & 94.06 & 91.1 & 1.02 \\
3 & 5 & 89 & 97.1 & 96 & 0.7 \\
4 & 23 & 87 & 97 & 93 & 0.38 \\
5 & 89.7 & 60 & 97 & 73 & 14 \\
6 & 8.8 & 84 & $\mathrm{NA}$ & $\mathrm{NA}$ & $\mathrm{NA}$ \\
\hline
\end{tabular}

CPAP, Continuous Positive Airway Pressure; AHI, Apnea Hypopnea Index; $\mathrm{SaO}_{2}$, Oxygen Saturation; ODI, Oxygen Desaturation Index; NA, Not Available

\section{Acknowledgements}

Funding: The capital health development research special project (2014-4-4087).

\section{Conflict of interest}

Author declares that there is no conflict of interest.

\section{References}

1. Thorpy MJ. Classification of sleep disorders. Neurotherapeutics. 2005;9(4):687-701.

2. Conrad Iber, Sonia AI, Andrew LC, et al. The new sleep scoring manual-The evidence behind the rules. Journal of Clinical Sleep Medicine. 2007;3(2): 107.

3. Oconnor GT, Caffo B, Newman AB, et al. Prospective study of sleep-disordered breathing and hypertension: the Sleep Heart Health Study. Am J Respir Crit Care Med. 2009;179(12):1159-1164.

4. Peppard PE, Young T, Palta M, et al. Prospective study of the association between sleep-disordered breathing and hypertension. $N$ Engl J Med. 2000;342(19):1378-1384.
5. Foster GD, Borradaile KE, Sanders MH, et al. A randomized study on the effect of weight loss on obstructive sleep apnea among obese patients with type 2 diabetes: the Sleep AHEAD study. Arch Intern Med. 2009;169(17):1619-1626.

6. Punjabi NM. The epidemiology of adult obstructive sleep apnea. Proc Am Thorac Soc. 2008;5(2):136-143.

7. Pien GW, Schwab RJ. Sleep disorders during pregnancy. Sleep. 2004;27(7):1405-1417.

8. Facco FL, Kramer J, Ho KH, et al. Sleep disturbances in pregnancy. Obstet Gynecol. 2010;115(1):77-83.

9. Sahota PK, Jain SS, Dhand R. Sleep disorders in pregnancy. Curr Opin Pulm Med. 2003;9(6):477-483.

10. Santiago JR, Nolledo MS, Kinzler W, et al. Sleep and sleep disorders in pregnancy. Ann Intern Med. 2001;134(5):396-408.

11. Bourjeily G, Raker CA, Chalhoub M, et al. Pregnancy and fetal outcomes of symptoms of sleep-disordered breathing. Eur Respir J. 2010;36(4):849-855.

12. Franklin KA, Holmgren PA, Jonsson F, et al. Snoring, pregnancy-induced hypertension, and growth retardation of the fetus. Chest. 2000;117(1):137-141. 
13. Facco FL, Grobman WA, Kramer J, et al. Self-reported short sleep duration and frequent snoring in pregnancy: impact on glucose metabolism. Am J Obstet Gynecol. 2010;203(2):142.e1-142e5.

14. Sullivan CE, Issa FG, Berthon-Jones M, et al. Reversal of obstructive sleep apnoea by continuous positive airway pressure applied through the nares. Lancet. 1981;1(8225):862-865.

15. Li XQ, Liu GL, Tang ZJ. Study the association of snoring at different stage in pregnancy with preeclampsia. Chin Clin J Obstet Gynecol. 2016;(4):333-336.

16. Facco FL, Ouyang DW, Zee PC, et al. Implications of sleep-disordered breathing in pregnancy. Am J Obstet Gynecol. 2014;210(6):559.e1-559. e6.

17. Resnick HE, Redline S, Shahar E, et al. Diabetes and sleep disturbances: findings from the Sleep Heart Health Study. Diabetes care. 2003;26(3):702-709.

18. Higgins N, Leong E, Park CS, et al. The Berlin Questionnaire for assessment of sleep disordered breathing risk in parturients and non pregnant women. Int J Obstet Anesth. 2011;20(1):22-25.
19. Barbe F, Duran-Cantolla J, Capote Fdela Pena M, et al. Long-term effect of continuous positive airway pressure in hypertensive patients with sleep apnea. Am J Respir Crit Care Med. 2010;181(7):718-726.

20. Duran-Cantolla J, Aizpuru F, Montserrat JM, et al. Continuous positive airway pressure as treatment for systemic hypertension in people with obstructive sleep apnoea: randomised controlled trial. $B M J$ 2010;341:c5991.

21. Haentjens P, Van Meerhaeghe A, Moscariello A, et al. The impact of continuous positive airway pressure on blood pressure in patients with obstructive sleep apnea syndrome: evidence from a meta-analysis of placebo-controlled randomized trials. Arch Intern Med. 2007;167(8):757-764

22. Marin JM, Carrizo SJ, Vicente E, et al. Long-term cardiovascular outcomes in men with obstructive sleep apnoea-hypopnoea with or without treatment with continuous positive airway pressure: an observational study. Lancet. 2005;365(9464):1046-1053. 